\section{P53 ACUTE WEST NILE VIRUS INFECTION IN AN SLE PATIENT - DIAGNOSTIC AND THERAPEUTIC CHALLENGES}

${ }^{1}$ Pavlos Tsakiridis, ${ }^{2}$ Lambros Athanassiou, ${ }^{1}$ Eirini Devetzi, ${ }^{1}$ Maria Mavroudi, 'Marina Gatsiou, 'Dimitrios Pantelidis, 'Aikaterini Tzanavari, 'Panagiotis Athanassiou. ${ }^{1}$ Dept. of Rheumatology, St. Paul's Hospital, Thessaloniki; 'First Dept. of Medicine, Asclepeion Hospital, Voula, Athens, Greece

10.1136/lupus-2020-eurolupus. 100

Background Systemic Lupus Erythematosus (SLE) patients are known to be prone to infections. In particular differential diagnosis between an acute infection or a disease flare should be performed in any SLE patient with fever or recurrent fever and signs of acute disease. Differential diagnosis becomes even more difficult in an SLE patient with central nervous system (CNS) involvement. The aim was to describe an SLE patient with CNS involvement and acute West Nile virus infection.

Methods A female patient was diagnosed with SLE at the age of 32 with a light-sensitive face eruption, hair loss, fatigue, arthralgias, ANA 1/320 (+) and anti-dsDNA (+). At the age of 45 she developed CNS involvement with epileptic seizures, dysarthria, memory loss, concentration difficulties and an abnormal EEG. At the age of 53 she had an acute SLE flare and pulse cyclophosphamide iv was administered. Thereafter rituximab was given followed by hydroxychloroquine and prednisolone $10 \mathrm{mg} / \mathrm{d}$. At the age of 54 years 3 months after the third rituximab cycle, while on therapy with hydroxychloroquine and prednisolone she developed diarrhea, vomiting and fever up to $40^{\circ} \mathrm{C}$ not responding to antipyretics. She presented to the emergency department with deteriorating renal function, fever and confusion.

Results A brain MRI showed meningeal thickening and a lumbar puncture was performed. The diagnostic evaluation of the fluid aspirated showed a recent infection with the West Nile virus with $\operatorname{IgM}(+++)$ in serum and $\operatorname{IgM}(+)$ in the cerebrospinal fluid. Two weeks later the patient had improved, was oriented in place and time and had no focal neurological signs.

Conclusions Patients with SLE are prone to infection, especially if they are on long-standing treatment with steroids. Whenever they present with signs of acute disease they should be carefully evaluated for the presence of an acute infection, as infections demand a different therapeutic approach to a disease flare. A patient with CNS involvement demands even more careful and extensive evaluation. The presence of West Nile virus in Europe in recent years along with other mosquito-borne viruses have created new diagnostic and therapeutic challenges in the management of immunosuppressed patients, as was the case in the patient presented herein.

\section{P54 INCIDENCE OF SKIN CANCER IN SYSTEMIC LUPUS ERYTHEMATOSUS COMPARED WITH SYSTEMIC SCLEROSIS AND GENERAL POPULATION}

\footnotetext{
${ }^{1}$ Ilaria Cavazzana, ${ }^{2,3}$ Marta Fusano, ${ }^{1,3}$ Micaela Fredi, ${ }^{2,3}$ Piergiacomo Calzavara-Pinton,

${ }^{1,3}$ Franco Franceschini. 'Rheumatology and Clinical Immunology Unit, ASST Spedali Civili Brescia, Brescia; ${ }^{2}$ Dermatology Unit, ASST Spedali Civili Brescia, Brescia; ${ }^{3}$ Dept. of Clinical and Experimental Sciences, University of Brescia, Brescia, Italy
}

10.1136/lupus-2020-eurolupus. 101
Background/Purpose This is a prospective study analysed the incidence of skin cancer (SC) (melanoma and non-melanoma SC) in 90 adult patients affected by Systemic Lupus Erythematosus (SLE), followed-up in one single Rheumatological Center, compared with 54 patients affected by Systemic Sclerosis (SSc) and 90 sex- and age-matched 90 control subject.

Methods In a period between February and July 2019, every patient underwent a complete dermatological evaluation and filled out a questionnaire regarding their personal or family history of SC, the presence of different risk factors of SC and the occurrence of photosensitivity.

Results 90 SLE patients $(96,7 \%$ female, mean age: 44 years; range: 18-78) showed photosensitivity in $60 \%$

$63 \%$ of patients avoided sun exposure at every hour of the day, $80 \%$ used photoprotection and 28\% referred systemic worsening of SLE features after sun exposure. No new onset skin cancer was diagnosed.

Three SLE patients referred a history of SC (1 basalioma, 1 melanoma, 1 multiple actinic lesions) onset after the SLE diagnosis. Patients with skin cancer (SC+) didn't show any differences compared with patients without skin cancer (SC-) except for more frequent photodamage features (p: 0.032) and less frequent photosensitivity (0.031).

SLE patients more frequently showed photosensitivity $(\mathrm{p}<0.0001)$, photoprotection $(\mathrm{p}<0.0001)$, disease worsening and skin worsening after sun exposure (p: 0.033 and 0.002, respectively) compared with SSc cases. No differences in past history of SC was evident between groups.

Comparing SLE with age-, sex- and phototype-matched control cohort, SLE patients showed a lower rate of past history of basalioma skin cancer (p: 0.013), lower rate of photodamage (0.027) and higher rate of photosensitivity $(\mathrm{p}<0.0001)$.

Conclusions SLE patients showed a significant lower rate of skin cancer, despite a higher rate of photosensitivity, compared with control cohort. This data could be due to a strict and continued photoprotection.

\section{P55 INFLUENCE OF DIETARY FIBRE AND SHORT-CHAIN FATTY ACIDS ON THE PATHOGENESIS OF SYSTEMIC LUPUS ERYTHEMATOSUS}

Alexandra Eichhorst, Anna-Lena Schäfer, Reinhard Edmund Voll, Nina Chevalier. Dept. of Rheumatology and Clinical Immunology, Freiburg University, Medical Centre, Freiburg, Germany

\subsection{6/lupus-2020-eurolupus. 102}

Background/Purpose In Westernized nations, the incidence of autoimmune and inflammatory diseases has dramatically risen over the past few decades. Environmental influences and their interplay with genetic risk factors have been suggested as important contributors to the rapid epidemic progress. Among environmental influences, Western diet - high in fat, sugar and salt - has been postulated as important risk, while beneficial effects were described for dietary fibre and their bacterial fermentation products, short chain fatty acids (SCFA). In SLE, their impact remains largely unexplored and was addressed in this study.

Methods Lupus prone NZB/WF1 mice were fed a low- (LFD) versus high-fibre diet (HFD) from the age of 3 weeks and over the course of the whole experiment. Determined were 